\title{
R-Parity violation in a SUSY GUT model and radiative neutrino masses
}

\author{
Joe Sato*† \\ Department of Physics, Faculty of Science, Saitama University, Saitama, 338-8570, \\ Japan \\ E-mail: joe@phy.saitama-u.ac.jp
}

ABstract: Within the framework of an SU(5) SUSY GUT model, a mechanism which effectively induces $R$-parity-violating terms below the unification energy scale $M_{X}$ is proposed. The model has matter fields $\overline{5}_{L(+)}+10_{L(-)}$ and Higgs fields $H_{(-)}$and $\bar{H}_{(+)}$in addition to the ordinary Higgs fields $H_{(+)}$and $\bar{H}_{(-)}$which contribute to the Yukawa interactions, where $( \pm)$ denote the transformation properties under a discrete symmetry $\mathrm{Z}_{2}$. The $\mathrm{Z}_{2}$ symmetry is only broken by the $\mu$-term $\bar{H}_{(+)} H_{(-)}$softly, so that the $\overline{5}_{(+)} \leftrightarrow \bar{H}_{(+)} \leftrightarrow \bar{H}_{(-)}$mixing appears at $\mu<m_{S B}$, and $R$-parity violating terms $\overline{5}_{L} \overline{5}_{L} 10_{L}$ are effectively induced from the Yukawa interactions $\bar{H}_{(-)} \overline{5}_{L(+)} 10_{L(-)}$, i.e. the effective coupling constants $\lambda_{i j k}$ of $\nu_{L i} e_{L j} e_{R k}^{c}$ and $\nu_{L i} d_{R j}^{c} d_{L k}$ are proportional to the mass matrices $\left(M_{e}^{*}\right)_{j k}$ and $\left(M_{d}^{\dagger}\right)_{j k}$, respectively. The parameter regions which are harmless for the proton decay are investigated. Possible forms of the radiatively induced neutrino mass matrix are also investigated.

\section{1. introduction}

The origin of the neutrino mass generation is still a mysterious problem in the unified understanding of the quarks and leptons. As the origin, from the standpoint of a grand unification theory (GUT), currently, the idea of the so-call seesaw mechanism[i $[\overline{2}]$ is influential. On the other hand, an alternative idea that the neutrino masses are radiatively induced is still attractive. As an example of such a model, the Zee model [3i]1] is well known. Regrettably, the original Zee model is not on the framework of GUT. A possible idea to embed the Zee model into GUTs is to identify the Zee scalar $h^{+}$as the slepton $\widetilde{e}_{R}$ in an $R$-parity-violating supersymmetric (SUSY) model [i] SUSY models with $R$-parity violation are incompatible with a GUT scenario, because the

${ }^{*}$ Speaker.

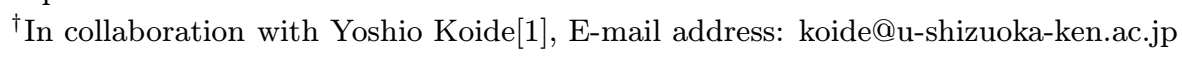


$R$-parity-violating interactions induce proton decay $\left[\overline{5}_{1}^{1}, \overline{6_{i}}\right]$. By the way, there is another problem in a GUT scenario, i.e. how to give doublet-triplet splitting in SU(5) 5-plet Higgs fields. There are many ideas to solve this problem $\left[\bar{i}_{1}\right]$. Although these mechanisms are very attractive, in the present paper, we will take another choice, that is, fine tuning of parameters: we consider a possibility that a mechanism which provides the doublet-triplet splitting gives a suppression of the $R$-parity violating terms with baryon number violation while it gives visible contributions of the doublet component to the low energy phenomena

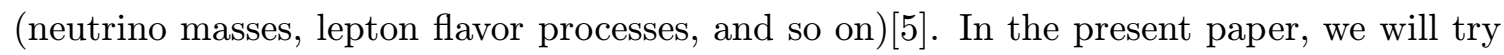
to give an example of such a scenario.

In the present paper, in order to suppress the dim-5 proton decay, a discrete symmetry $\mathrm{Z}_{2}$ is introduced. The essential idea is as follows: we consider matter fields $\overline{5}_{L(+)}+10_{L((-)}$ of SU(5) and two types of SU(5) 5-plet and $\overline{5}$-plet Higgs fields $H_{( \pm)}$and $\bar{H}_{( \pm)}$, where $\left( \pm\right.$ ) denote the transformation properties under a discrete symmetry $\mathrm{Z}_{2}$ (we will call it " $\mathrm{Z}_{2}$-parity" hereafter). The superpotential in the present model is given by

$$
W=W_{Y}+W_{H}+W_{\text {mix }},
$$

where $W_{Y}$ denotes Yukawa interactions

$$
W_{Y}=\sum_{i, j}\left(Y_{u}\right)_{i j} H_{(+)} 10_{L(-) i} 10_{L(-) j}+\sum_{i, j}\left(Y_{d}\right)_{i j} \bar{H}_{(-)} \overline{5}_{L(+) i} 10_{L(-) j} .
$$

Under the discrete symmetry $\mathrm{Z}_{2}, R$-parity violating terms $\overline{5}_{L(+)} \overline{5}_{L(+)} 10_{(-)}$are exactly forbidden. The discrete symmetry $\mathrm{Z}_{2}$ is softly violated only by the following $\mu$-terms

$$
W_{H}=\bar{H}_{(+)}\left(m_{+}+g_{+} \Phi\right) H_{(+)}+\bar{H}_{(-)}\left(m_{-}+g_{-} \Phi\right) H_{(-)}+m_{S B} \bar{H}_{(+)} H_{(-)},
$$

where $\Phi$ is an $\mathrm{SU}(5)$ 24-plet Higgs field with the vacuum expectation value (VEV) $\langle\Phi\rangle=$ $v_{24} \operatorname{diag}(2,2,2,-3,-3)$, and it has been introduced in order to give doublet-triplet splittings in the $\mathrm{SU}(5)$ 5- and 5-plets Higgs fields at an energy scale $\mu<M_{X}\left(M_{X}\right.$ is an $\mathrm{SU}(5)$ unification scale). The $\mathrm{Z}_{2}$-parity is violated only by ${ }^{1}$ the term $\bar{H}_{(+)} H_{(-)}$. Note that $H_{(-)}$ and $\bar{H}_{(+)}$in the $m_{S B}$-term do not contribute to the Yukawa interaction (1.2) directly, so that proton decay via the dimension-5 operator is suppressed in the limit of $m_{S B} \rightarrow 0$. (A similar idea, but without $\mathrm{Z}_{2}$ symmetry, has been proposed by Babu and Barr [i.8].) The terms $W_{\text {mix }}$ have been introduced in order to bring the $\bar{H}_{(+)} \leftrightarrow \overline{5}_{(+)}$mixing:

$$
W_{\text {mix }}=\sum_{i} \overline{5}_{L(+) i}\left(b_{i} m_{5}+c_{i} g_{5} \Phi\right) H_{(+)}
$$

where $\sum_{i}\left|b_{i}\right|^{2}=\sum_{i}\left|c_{i}\right|^{2}=1$. At the energy scale $\mu<M_{X}$, the terms $W_{H}+W_{\text {mix }}$ are effectively given by

$$
\begin{aligned}
W_{H}+W_{\text {mix }}= & \sum_{a=2,3} m_{+}^{(a)}\left[\bar{H}_{(+)}^{(a)} \cos \alpha^{(a)}+\sum_{i} d_{i} \overline{5}_{L(+) i}^{(a)} \sin \alpha^{(a)}\right] H_{(+)}^{(a)} \\
& +\sum_{a=2,3} m_{-}^{(a)} \bar{H}_{(-)}^{(a)} H_{(-)}^{(a)}+m_{S B} \sum_{a=2,3} \bar{H}_{(+)}^{(a)} H_{(-)}^{(a)}
\end{aligned}
$$

\footnotetext{
${ }^{1}$ The $\mathrm{Z}_{2}$ symmetry can softly violated not only by the term $\bar{H}_{(+)} H_{(-)}$, but also by terms $\bar{H}_{(-)} H_{(+)}$ and $\overline{5}_{L(+) 1} H_{(-)}$. However, in the present scenario, the existence of $\bar{H}_{(+)} H_{(-)}$is essential. The details are discussed in Appendix A of
} 
where $\sum_{i}\left|d_{i}\right|^{2}=1$, the index (a) denotes that the fields with (2) and (3) are doublet and triplet components of $\mathrm{SU}(5) \rightarrow \mathrm{SU}(2) \times \mathrm{SU}(3)$, respectively, and

$$
\begin{array}{cl}
m_{+}^{(2)} \cos \alpha^{(2)}=m_{+}-3 g_{+} v_{24}, & m_{+}^{(3)} \cos \alpha^{(3)}=m_{+}+2 g_{+} v_{24}, \\
m_{+}^{(2)} \sin \alpha^{(2)} d_{i}=m_{5} b_{i}-3 g_{5} v_{24} c_{i}, & m_{+}^{(3)} \sin \alpha^{(3)} d_{i}=m_{5} b_{i}+2 g_{5} v_{24} c_{i}, \\
m_{-}^{(2)}=m_{-}-3 g_{-} v_{24}, \quad m_{-}^{(3)}=m_{-}+2 g_{-} v_{24} .
\end{array}
$$

Therefore, the $m_{S B}$-term together with $m_{+} \sin \alpha$-term induces the $\bar{H}_{(-)} \leftrightarrow \overline{5}_{L(+)}$ mixing, so that the $R$-parity violating terms $\overline{5}_{L} \overline{5}_{L} 10_{L}$ are generated from the Yukawa interactions $\bar{H}_{(-)} \overline{5}_{L(+)} 10_{L(-)}$. The coupling constants $\lambda_{i j k}$ of $\overline{5}_{i} \overline{5}_{j} 10_{k}$ will be proportional to the charged lepton mass matrix $\left(M_{e}^{*}\right)_{j k}$ or down-quark mass matrix $\left(M_{d}^{\dagger}\right)_{j k}$. (The details are discussed in the next section 2.) As we demonstrate in Sec. 2, we can show that the mixing $\overline{5}_{L(+)} \leftrightarrow$ $\bar{H}_{(-)}$is negligibly small for the colored sector, while it is sizable for $\mathrm{SU}(2)$-doublet sector.

The parameters in the present model need fine-tuning. For example, we will find that a large value of $m_{S B}$ is not acceptable, because for such a large value of $m_{S B}$ the proton decay due to the dimension five operator becomes visible. On the other hand, we will find that a smaller value of $m_{S B}$ leads to a small bottom quark mass, so that a small value of $m_{S B}$ is not acceptable. We will take $m_{S B} \sim 10^{14} \mathrm{GeV}$. In Sec. 3, we will investigate a possible form of the radiatively induced neutrino mass matrix due to the $R$-parity violation term $\overline{5}_{L} \overline{5}_{L} 10_{L}$. The radiatively induced neutrino mass matrix $M_{\nu}^{\text {rad }}$ will be expressed by the sum of two rank-1 matrices. On the other hand, we also have contributions $M_{\tilde{\nu}}$ from VEVs $\left\langle\tilde{\nu}_{i}\right\rangle$ of the sneutrinos to the neutrino mass matrix $M_{\nu}$. Finally, Sec. 4 will be devoted to the summary.

\section{2. $\bar{H}_{(-)}-\overline{5}_{(+)}$mixing}

In order to suppress the proton decay, we want to take $m_{+}^{(2)} \sim M_{W}$ with a sizable $\alpha^{(2)}$, but

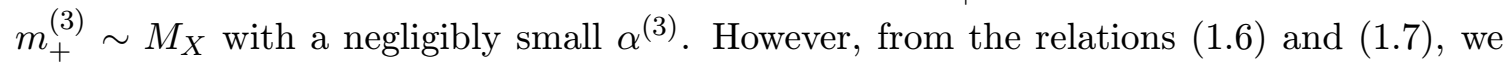
obtain the relation

$$
d_{i} \tan \alpha^{(3)}=\frac{m_{5} b_{i}+2 g_{5} v_{24} c_{i}}{m_{+}+2 g_{+} v_{24}}=\frac{m_{+}^{(2)} \sin \alpha^{(2)} d_{i}+5 g_{5} v_{24} c_{i}}{m_{+}^{(2)} \cos \alpha^{(2)}+5 g_{+} v_{24}} .
$$

The requirement $\left|\alpha^{(3)}\right| \leq M_{W} / M_{X}$ leads to the constraint $\left|g_{5}\right| \leq M_{W} / M_{X}$ for $\left|g_{+}\right| \sim 1$. We do not like to introduce such a small dimensionless parameter $g_{5}$. Therefore, for simplicity, we will put $g_{5}=0$ hereafter. Then, without loss of generality, we can put

$$
\overline{5}_{L(+) 1}^{\prime}=\sum_{i} b_{i} \overline{5}_{L(+) i}
$$

where $\overline{5}_{L(+) 1}^{\prime}$ does not mean the observed first generation particle. (Hereafter, for convenience, we denote $\overline{5}_{L(+) 1}^{\prime}$ as $\overline{5}_{L(+) 1}$ simply. The effective parameters $m_{+}^{(a)}, m_{-}^{(a)}$ and $\alpha^{(a)}$ 
are given as follows:

$$
\begin{array}{cl}
m_{+}^{(2)}=\sqrt{\left(m_{+}-3 g_{+} v_{24}\right)^{2}+m_{5}^{2}}, & m_{+}^{(3)}=\sqrt{\left(m_{+}+2 g_{+} v_{24}\right)^{2}+m_{5}^{2}}, \\
m_{-}^{(2)}=m_{-}-3 g_{-} v_{24}, & m_{-}^{(3)}=m_{-}+2 g_{-} v_{24}, \\
\tan \alpha^{(2)}=\frac{m_{5}}{m_{+}-3 g_{+} v_{24}} \simeq \frac{m_{5}}{m_{+}^{(2)}}, & \tan \alpha^{(3)}=\frac{m_{5}}{m_{+}+2 g_{+} v_{24}} \simeq \frac{m_{5}}{m_{+}^{(3)}} .
\end{array}
$$

We will take

$$
\begin{aligned}
m_{+}^{(2)} & \sim M_{W}, & & m_{+}^{(3)} \sim M_{X}, \\
m_{-}^{(2)} & \sim M_{I}, & & m_{-}^{(3)} \sim M_{X}, \\
\tan \alpha^{(2)} & \sim \frac{m_{5}}{M_{W}}, & & \tan \alpha^{(3)} \sim \frac{m_{5}}{M_{X}},
\end{aligned}
$$

where $M_{I} \sim 10^{14} \mathrm{GeV}$ and $m_{5} \sim 10^{1} \mathrm{GeV}$ as we state later. The mass matrix in the basis of $\left(\bar{H}_{(-)}, \bar{H}_{(+)}, \overline{5}_{L(+) 1}\right)$ and $\left(H_{(+)}, H_{(-)}\right)$is given by

$$
M=\left(\begin{array}{cc}
0 & m_{-} \\
m_{+} \cos \alpha & m_{S B} \\
m_{+} \sin \alpha & 0
\end{array}\right) .
$$

Here and hereafter, for simplicity, we drop the index $(a)$. The mass matrix $(\overline{2} . \overline{2} \cdot)$ is diagonalized as

$$
\bar{U}^{\dagger} M U=D \equiv\left(\begin{array}{cc}
m_{1} & 0 \\
0 & m_{2} \\
0 & 0
\end{array}\right) .
$$

Note that the matter field $\overline{5}_{L 1}^{\prime}$ is still massless, and also note that it is not in the eigenstate of the $\mathrm{Z}_{2}$ parity.

The mixing matrix $U$ is easily obtained from the diagonalization of

$$
M^{\dagger} M=\left(\begin{array}{cc}
\left|m_{+}\right|^{2} & m_{S B} m_{+}^{*} \cos \alpha \\
m_{S B}^{*} m_{+} \cos \alpha & \left|m_{S B}\right|^{2}+\left|m_{-}\right|^{2}
\end{array}\right) .
$$

For real $m_{1}, m_{S B}$ and $m_{ \pm}$, we obtain

$$
\begin{gathered}
U=\left(\begin{array}{cc}
\cos \theta_{u} & \sin \theta_{u} \\
-\sin \theta_{u} & \cos \theta_{u}
\end{array}\right), \\
\tan 2 \theta_{u}=\frac{2 m_{S B} m_{+} \cos \alpha}{m_{S B}^{2}+m_{-}^{2}-m_{+}^{2}}, \\
m_{1}^{2}=\frac{1}{2}\left(m_{S B}^{2}+m_{+}^{2}+m_{-}^{2}\right)-\frac{1}{2} Q, \\
m_{2}^{2}=\frac{1}{2}\left(m_{S B}^{2}+m_{+}^{2}+m_{-}^{2}\right)+\frac{1}{2} Q,
\end{gathered}
$$

where

$$
Q=\left(m_{S B}^{2}-m_{+}^{2}+m_{-}^{2}\right) \cos 2 \theta_{u}+2 m_{S B} m_{+} \cos \alpha \sin 2 \theta_{u}
$$


When we define

$$
\begin{aligned}
& A \equiv m_{S B}^{2}-m_{+}^{2}+m_{-}^{2}, \quad B \equiv 2 m_{S B} m_{+} \cos \alpha, \\
& \cos 2 \theta_{u}=\frac{A}{\sqrt{A^{2}+B^{2}}}, \quad \sin 2 \theta_{u}=\frac{B}{\sqrt{A^{2}+B^{2}}},
\end{aligned}
$$

the quantity $Q$ is given by

$$
Q=\sqrt{A^{2}+B^{2}}=\sqrt{\left[m_{S B}^{2}+\left(m_{+}-m_{-}\right)^{2}\right]\left[m_{S B}^{2}+\left(m_{+}+m_{-}\right)^{2}\right]-4 m_{S B}^{2} m_{+}^{2} \sin ^{2} \alpha} .
$$

The rotation $\bar{U}$ is also obtained from the diagonalization of

$$
M M^{\dagger}=\left(\begin{array}{ccc}
m_{-}^{2} & m_{S B} m_{-} & 0 \\
m_{S B} m_{-} & m_{S B}^{2}+m_{+}^{2} \cos ^{2} \alpha & m_{+}^{2} \cos \alpha \sin \alpha \\
0 & m_{+}^{2} \cos \alpha \sin \alpha & m_{+}^{2} \sin ^{2} \alpha
\end{array}\right)
$$

The mixing matrix elements $\bar{U}_{i 3}$ are easily obtain as follows:

$$
\bar{U}_{13}=\frac{1}{N_{3}} m_{S B} \sin \alpha, \bar{U}_{23}=-\frac{1}{N_{3}} m_{-} \sin \alpha, \bar{U}_{33}=\frac{1}{N_{3}} m_{-} \cos \alpha,
$$

where

$$
N_{3}^{2}=-m_{-}^{2}+m_{S B}^{2} \sin ^{2} \alpha
$$

Other matrix elements are obtained as follows: We express the mixing matrix $\bar{U}$ as

$$
\bar{U}=\left(\begin{array}{ccc}
c_{13} c_{12} & c_{13} s_{12} & s_{13} \\
-c_{23} s_{12}-s_{23} c_{12} s_{13} & c_{23} c_{12}-s_{23} s_{12} s_{13} & s_{23} c_{13} \\
s_{23} s_{12}-c_{23} c_{12} s_{13} & -s_{23} c_{12}-c_{23} s_{12} s_{13} & c_{23} c_{13}
\end{array}\right),
$$

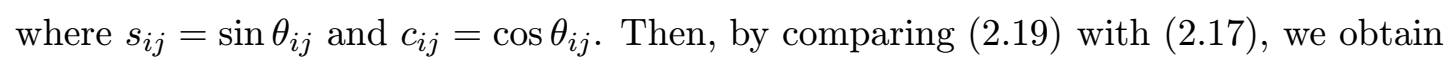

$$
\begin{gathered}
s_{13}=\bar{U}_{13}=\frac{m_{S B} \sin \alpha}{\sqrt{m_{-}^{2}+m_{S B}^{2} \sin ^{2} \alpha}}, \quad c_{13}=\frac{1}{\sqrt{1+\left(m_{S B} / m_{-}\right)^{2} \sin ^{2} \alpha}}, \\
s_{23}=\frac{\bar{U}_{23}}{c_{13}}=-\sin \alpha, \quad c_{23}=\cos \alpha .
\end{gathered}
$$

By using the relation $\left(M^{\dagger} M\right)_{11}=\bar{U}_{11} \bar{U}_{11}\left(m_{1}^{\prime}\right)^{2}+\bar{U}_{12} \bar{U}_{12}\left(m_{2}^{\prime}\right)^{2}$, the mixing angle $\theta_{12}$ is obtained as follows:

$$
\begin{aligned}
\cos 2 \theta_{12} & =\frac{1}{m_{2}^{2}-m_{1}^{2}}\left[m_{1}^{2}+m_{2}^{2}-2 \frac{m_{-}^{2}}{c_{13}^{2}}\right]=\frac{1}{Q}\left(m_{S B}^{2}+m_{+}^{2}-m_{-}^{2}-2 m_{S B}^{2} \sin ^{2} \alpha\right) \\
& =-\frac{m_{-}^{2}-m_{2}^{2} \cos 2 \alpha-m_{+}^{2}}{\sqrt{m_{-}^{4}+2\left(m_{2}^{2}-m_{+}^{2}\right) m_{-}^{2}+m_{2}^{4}+2 m_{2}^{2} m_{+}^{2} \cos 2 \alpha+m_{+}^{4}}}
\end{aligned}
$$

Note that $\cos 2 \theta_{12} \simeq-1$ for $m_{-}^{2} \gg m_{S B}^{2}, m_{+}^{2}$, so that $\theta_{12} \simeq \pi / 2$. 
Since the physical fields $\left(\bar{H}_{1}, \bar{H}_{2}, \overline{5}_{L 1}^{\prime}, \overline{5}_{L 2}^{\prime}, \overline{5}_{L 3}^{\prime}\right)$ are given by

$$
\left(\begin{array}{c}
\bar{H}_{(-)} \\
\bar{H}_{(+)} \\
\overline{5}_{L(+) 1} \\
\overline{5}_{L(+) 2} \\
\overline{5}_{L(+) 3}
\end{array}\right)=\left(\begin{array}{ccccc}
\bar{U}_{11} & \bar{U}_{12} & \bar{U}_{13} & 0 & 0 \\
\bar{U}_{21} & \bar{U}_{22} & \bar{U}_{23} & 0 & 0 \\
\bar{U}_{31} & \bar{U}_{32} & \bar{U}_{33} & 0 & 0 \\
0 & 0 & 0 & 1 & 0 \\
0 & 0 & 0 & 0 & 1
\end{array}\right)\left(\begin{array}{c}
\bar{H}_{1} \\
\bar{H}_{2} \\
\overline{5}_{L 1}^{\prime} \\
\overline{5}_{L 2}^{\prime} \\
\overline{5}_{L 3}^{\prime}
\end{array}\right),
$$

the Yukawa interactions $\bar{H}_{(-)} \overline{5}_{L(+)} 10_{L(-)}$ lead to the effective Yukawa interactions at a low energy scale

$$
\left(Y_{d}\right)_{i j} \bar{H}_{1}\left[\delta_{i 1}\left(\bar{U}_{11} \bar{U}_{33}-\bar{U}_{13} \bar{U}_{31}\right) \overline{5}_{L 1}^{\prime}+\bar{U}_{11}\left(\delta_{i 2} \overline{5}_{L 2}^{\prime}+\delta_{i 3} \overline{5}_{L 3}^{\prime}\right)\right] 10_{L(-) j},
$$

and the induced $R$-parity violating terms

$$
\left(Y_{d}\right)_{i j} \bar{U}_{13} \overline{5}_{L 1}^{\prime}\left(\delta_{i 1} \bar{U}_{33} \overline{5}_{L 1}^{\prime}+\delta_{i 2} \overline{5}_{L 2}^{\prime}+\delta_{i 3} \overline{5}_{L 3}^{\prime}\right) 10_{L(-) j}
$$

where we have assumed that $\left|m_{1}\right|<<\left|m_{2}\right|$, i.e. the Higgs field surviving at a low energy scale is not $\bar{H}_{2}$, but $\bar{H}_{1}$.

The effective Yukawa interactions $(\overline{2} . \overline{2} \overline{4})$ give the fermion mass matrices

$$
\begin{gathered}
\left(M_{e}^{*}\right)_{i j}= \begin{cases}\left(\bar{U}_{11}^{(2)} \bar{U}_{33}^{(2)}-\bar{U}_{13}^{(2)} \bar{U}_{31}^{(2)}\right)\left(Y_{d}\right)_{i j} v_{d}=\bar{U}_{22}^{(2) *}\left(Y_{d}\right)_{i j} v_{d} & \text { for } i=1, \\
\bar{U}_{11}^{(2)}\left(Y_{d}\right)_{i j} v_{d} & \text { for } i=2,3,\end{cases} \\
\left(M_{d}^{\dagger}\right)_{i j}= \begin{cases}\left(\bar{U}_{11}^{(2)} \bar{U}_{33}^{(3)}-\bar{U}_{13}^{(3)} \bar{U}_{31}^{(2)}\right)\left(Y_{d}\right)_{i j} v_{d} & \text { for } i=1, \\
\bar{U}_{11}^{(2)}\left(Y_{d}\right)_{i j} v_{d} & \text { for } i=2,3,\end{cases}
\end{gathered}
$$

where $v_{d}=\left\langle\bar{H}_{1}\right\rangle$, and, in $\left(\overline{2}_{2} \cdot \overline{2} \overline{6}\right)$, we have used the general formula $U_{i k} U_{j l}-U_{i l} U_{j k}=$ $\varepsilon_{i j m} \varepsilon_{k l n} U_{m n}^{*}$ for an arbitrary $3 \times 3$ unitary matrix $U$. Note that in the present model, the relation $M_{d}=M_{e}^{T}$ does not hold.

From the $R$-parity violating terms $(2.25)$, we obtain coefficients $\lambda_{i j k}^{(2,2)}, \lambda_{i j k}^{(2,3)}, \lambda_{i j k}^{(3,2)}$ and $\lambda_{i j k}^{(3,3)}$, which are the coefficients of the interactions $\left(\nu_{L 1} e_{L i}-e_{L 1} \nu_{L i}\right) e_{R j}^{c},\left(\nu_{L 1} d_{R i}^{c} d_{L j}-\right.$ $\left.e_{L 1} d_{R i}^{c} u_{L j}\right),\left(d_{R 1}^{c} e_{L i} u_{L j}-d_{R 1}^{c} \nu_{L i} d_{L j}\right)$, and $\varepsilon_{\alpha \beta \gamma} d_{R 1}^{c \alpha} d_{R i}^{c \beta} u_{R j}^{c \gamma}$, respectively, as follows:

$$
\begin{aligned}
& \lambda_{11 j}^{(2,2)}=0, \quad \lambda_{1 i j}^{(2,2)}=\kappa\left(M_{e}^{*}\right)_{i j} / v_{d} \quad(i=2,3), \\
& \lambda_{11 j}^{(2,3)}=\frac{\kappa\left(M_{d}^{\dagger}\right)_{1 j} / v_{d}}{1-\xi \kappa \bar{U}_{31}^{(2)} / \bar{U}_{33}^{(3)}}, \quad \lambda_{1 i j}^{(2,3)}=\kappa\left(M_{d}^{\dagger}\right)_{i j} / v_{d} \quad(i=2,3), \\
& \lambda_{11 j}^{(3,2)}=\frac{\xi \kappa\left(M_{e}^{*}\right)_{1 j} / v_{d}}{1-\kappa \bar{U}_{31}^{(2)} / \bar{U}_{33}^{(2)}}, \quad \lambda_{1 i j}^{(3,2)}=\xi \kappa\left(M_{e}^{*}\right)_{i j} / v_{d} \quad(i=2,3), \\
& \lambda_{11 j}^{(3,3)}=0, \quad \lambda_{1 i j}^{(3,3)}=\xi \kappa\left(M_{d}^{\dagger}\right)_{i j} / v_{d} \quad(i=2,3),
\end{aligned}
$$

where

$$
\kappa=\frac{\bar{U}_{13}^{(2)}}{\bar{U}_{11}^{(2)}}, \quad \xi=\frac{\bar{U}_{13}^{(3)}}{\bar{U}_{13}^{(2)}} .
$$




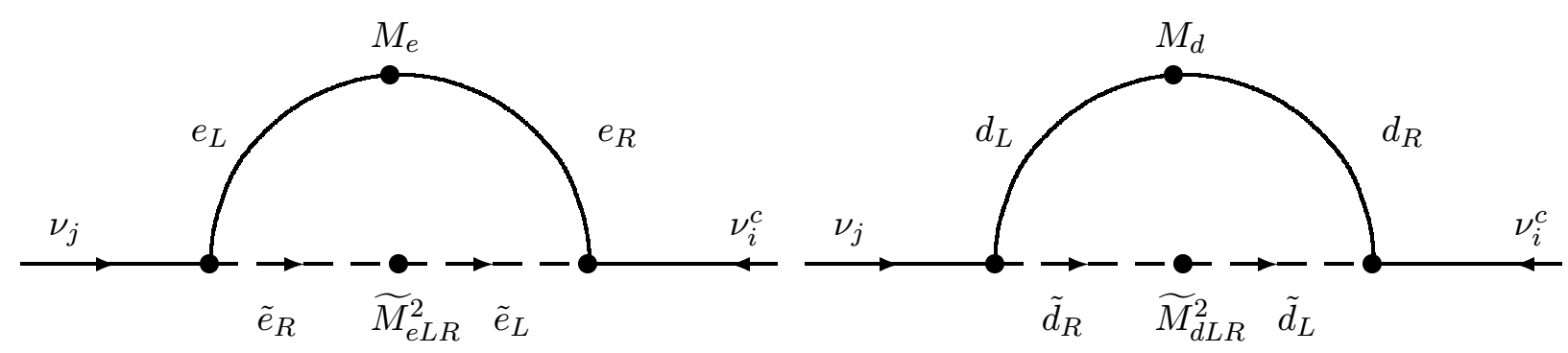

Figure 1: Radiative generation of neutrino Majorana mass

Note that the proton decay due to the exchange of squarks $\widetilde{d}_{i}$ is forbidden in the limit of $\xi \rightarrow 0$, while the radiatively-induced neutrino masses do not become zero even if $\xi \rightarrow 0$. To suppress the proton decay due to these couplings, $\kappa$ should be small while to have neutrino masses $\kappa$ should have a sufficient strength. In this respect we can show that $\kappa \sim O(0.1)$ is welcome. Also to suppress the proton decay through dimension 5 operators it is necessary that $m_{S B}$ must be small, though it is also bounded from below to make yukawa couplings be sufficiently large. [i]1,

\section{Radiatively induced neutrino mass matrix}

In a SUSY GUT scenario, there are many origins of the neutrino mass generations $[1 \overline{1} 0 \overline{0}]$. For example, the sneutrinos $\widetilde{\nu}_{i L}$ can have VEVs $\langle\widetilde{\nu}\rangle \neq 0$, and the neutrinos $\nu_{L i}$ acquire their masses thereby [i] $\left.{ }_{1}^{1} \overline{1}\right]$. In the present model, there is a $R$-parity violating bilinear term $\overline{5}_{L(+)} H_{(+)}$, while there is no $\bar{H}_{(-)} H_{(+)}$term (the so-called $\mu$-term). In the physical field

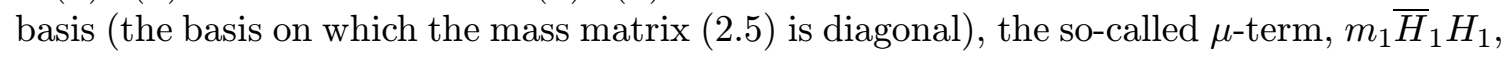
appears, while the $\overline{5}_{L} H_{1}$ term is absent. Therefore, in the present model, the sneutrinos cannot have VEVs $\left\langle\tilde{\nu}_{i}\right\rangle$ at tree level. The VEVs $\left\langle\tilde{\nu}_{i}\right\rangle \neq 0$ will appear only through the renormalization group equation (RGE) effect. The contribution highly depends on an explicit model of the SUSY symmetry breaking. Since the purpose of the present paper is to investigate a general structure of the radiative neutrino masses, for the moment, we confine ourselves to discussing possible forms of the radiative neutrino mass matrix .

The radiative neutrino mass matrix $M_{\nu}^{\text {rad }}$ is given by

$$
M_{\nu}^{r a d}=M_{\nu}^{e}+M_{\nu}^{d}
$$

where $M_{\nu}^{e}$ is generated by the interactions $\nu_{L} e_{L} \widetilde{e}_{R}^{c}$ and $\nu_{L} \widetilde{e}_{L} e_{R}^{c}$ (i.e. by the charged lepton loop) and $M_{\nu}^{d}$ is generated by $\nu_{L} d_{R}^{c} \widetilde{d}_{L}$ and $\nu_{L} \widetilde{d}_{R}^{c} d_{L}$ (i.e. by the down-quark loop). We assume that the contributions from Zee-type diagrams due to $\bar{H}^{+} \leftrightarrow \widetilde{e}_{R}^{+}$mixing is negligibly small because the term $\bar{H} \bar{H} 10_{L}$ must be not $\bar{H}_{1} \bar{H}_{1} 10_{L}$, but $\bar{H}_{1} \bar{H}_{2} 10_{L}$ (recall that only the field $\bar{H}_{1}$ has the VEV in the present model).

We consider the radiative diagram with $\left(\nu_{L}\right)_{j} \rightarrow\left(e_{R}\right)_{l}+\left(\widetilde{e}_{L}^{c}\right)_{n}$ and $\left(e_{L}\right)_{k}+\left(\widetilde{e}_{L}^{c}\right)_{m} \rightarrow$ $\left(\nu_{L}^{c}\right)_{i}$. The contributions $\left(M_{\nu}^{e}\right)_{i j}$ from the charged lepton loop are, except for the common 
factors, given as follows:

$$
\left(M_{\nu}^{e}\right)_{i j}=\left(\lambda_{1 k m} \delta_{i 1}-\lambda_{1 i m} \delta_{k 1}\right)\left(\lambda_{1 j l} \delta_{n 1}-\lambda_{1 n l} \delta_{j 1}\right)\left(M_{e}\right)_{k l}\left(\widetilde{M}_{e L R}^{2}\right)_{n m}+(i \leftrightarrow j),
$$

where $M_{e}$ and $\widetilde{M}_{e L R}^{2}$ are charged-lepton and charged-slepton- $L R$ mass matrices, respectively. Here and hereafter, we will drop the common factor in $\left(M_{\nu}^{r a d}\right)_{i j}$, because we have an interest only in the relative structure of the matrix elements $\left(M_{\nu}^{r a d}\right)_{i j}$. Since, as usual, we assume that the structure of $\widetilde{M}_{e L R}^{2}$ is proportional to that of $M_{e}$, we obtain

$$
\begin{aligned}
\left(M_{\nu}^{e}\right)_{i j}= & \lambda_{1 i m} \lambda_{1 j l}\left(M_{e}\right)_{1 l}\left(M_{e}\right)_{1 m}+\delta_{i 1} \delta_{j 1} \lambda_{1 k m} \lambda_{1 n l}\left(M_{e}\right)_{k l}\left(M_{e}\right)_{n m} \\
& -\delta_{i 1} \lambda_{1 j l} \lambda_{1 k m}\left(M_{e}\right)_{1 m}\left(M_{e}\right)_{k l}-\delta_{j 1} \lambda_{1 i l} \lambda_{1 k m}\left(M_{e}\right)_{1 m}\left(M_{e}\right)_{k l}
\end{aligned}
$$

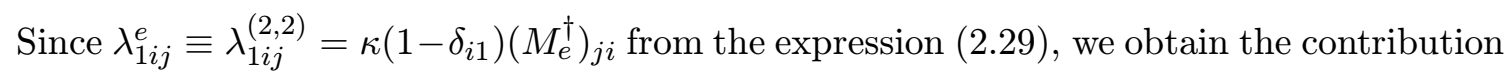
from the charged lepton loop:

$$
M_{\nu}^{e}=H_{e}^{T} S_{1} H_{e}-S_{1} H_{e} H_{e}-H_{e}^{T} H_{e}^{T} S_{1}+S_{1} \operatorname{Tr}\left(H_{e} H_{e}\right) .
$$

where we have dropped the common factor $\kappa$, and the Hermitian matrix $H_{e}$ and the rank-1 matrix $S_{1}$ are defined by

$$
\begin{gathered}
H_{e}=M_{e} M_{e}^{\dagger}, \\
S_{1}=\left(\begin{array}{lll}
1 & 0 & 0 \\
0 & 0 & 0 \\
0 & 0 & 0
\end{array}\right) .
\end{gathered}
$$

Similarly, we can obtain the contributions from the down-quark loop. From the expression (2.2.30i), we denote $\lambda_{1 i j}^{d} \equiv \lambda_{1 i j}^{(2,3)}$ as

$$
\lambda_{1 i j}^{d}=\kappa\left[\rho \delta_{1 i}+\left(1-\delta_{i 1}\right)\right]\left(M_{d}^{\dagger}\right)_{i j},
$$

where

$$
\rho=\frac{1}{1-\xi \kappa U_{31}^{(2)} / U_{33}^{(3)}} \simeq 1
$$

Then, we obtain

$$
M_{\nu}^{d}=S_{1} \operatorname{Tr}\left(H_{d} H_{d}\right)
$$

where

$$
H_{d}=M_{d}^{\dagger} M_{d} .
$$

Note that the result $\left(\overline{\underline{3}} . \bar{q}_{1}\right)$ is independent of the value of $\rho$.

The field $\overline{5}_{L(+) 1}$ defined in Eq. (2.2.2. $\left.\overline{2}_{1}^{\prime}\right)$ does not mean the observed first-generation field $\left(d^{c}, \nu, e\right)_{L}$ (and its SUSY partner). The forms of $M_{\nu}^{e}$ and $M_{\nu}^{d}$ on the general basis are given by

$$
\begin{gathered}
M_{\nu}^{e}=H_{e}^{T} S H_{e}-S H_{e} H_{e}-H_{e}^{T} H_{e}^{T} S+S \operatorname{Tr}\left(H_{e} H_{e}\right), \\
M_{\nu}^{d}=S \operatorname{Tr}\left(H_{d} H_{d}\right),
\end{gathered}
$$

where $S$ is an arbitrary rank-1 matrix $S=U_{5}^{T} S_{1} U_{5}$, which is given by the rebasing $\overline{5}_{i} \rightarrow$ $\overline{5}_{i}^{\prime}=\left(U_{5}^{\dagger} \overline{5}\right)_{i}$. 
It is convenient to investigate the form $M_{\nu}^{\text {rad }}$ on the basis on which the charged lepton mass matrix $M_{e}$ is diagonal: $H_{e}=D_{e}^{2}=\operatorname{diag}\left(m_{e 1}^{2}, m_{e 2}^{2}, m_{e 3}^{2}\right) \equiv \operatorname{diag}\left(m_{e}^{2}, m_{\mu}^{2}, m_{\tau}^{2}\right)$. Then, the matrix $M_{\nu}^{e}$ is given by

$$
\begin{aligned}
M_{\nu}^{e} & =\left(\begin{array}{ccc}
S_{11}\left(m_{e 2}^{4}+m_{e 3}^{4}\right) & S_{12}\left(m_{e 3}^{4}+m_{e 1}^{2} m_{e 2}^{2}\right) & S_{13}\left(m_{e 2}^{4}+m_{e 1}^{2} m_{e 3}^{2}\right) \\
S_{21}\left(m_{e 3}^{4}+m_{e 1}^{2} m_{e 2}^{2}\right) & S_{22}\left(m_{e 3}^{4}+m_{e 1}^{4}\right) & S_{23}\left(m_{e 1}^{4}+m_{e 2}^{2} m_{e 3}^{2}\right) \\
S_{31}\left(m_{e 2}^{4}+m_{e 1}^{2} m_{e 3}^{2}\right) & S_{32}\left(m_{e 1}^{4}+m_{e 2}^{2} m_{e 3}^{2}\right) & S_{33}\left(m_{e 1}^{4}+m_{e 2}^{4}\right)
\end{array}\right) \\
& \simeq m_{\tau}^{4}\left(\begin{array}{ccc}
S_{11} & S_{12} & 0 \\
S_{21} & S_{22} & 0 \\
0 & 0 & 0
\end{array}\right)=m_{\tau}^{4} P S P \\
P & =\operatorname{diag}(1,1,0)
\end{aligned}
$$

Therefore, we can express the neutrino mass matrix $M_{\nu}^{\text {rad }}$ as the following form:

$$
M_{\nu}^{r a d}=m_{0}\left(P S P+k^{2} S\right)
$$

where $k$ is given by $k \simeq\left(m_{b} / m_{\tau}\right)^{2}$ and $m_{0}$ will be given later [in Eq. (3.18i)]. The matrix $S$ is a rank-1 matrix, so that $P S P$ is also rank-1 matrix. In other words, the radiative neutrino mass matrix $M_{\nu}^{r a d}$ has the following form by assuming $\left(U_{5}\right)_{1 e} \sim O(\epsilon),\left(U_{5}\right)_{1 \mu},\left(U_{5}\right)_{1 \tau} \sim O(1)$

$$
M_{\nu}^{r a d}=m_{0}\left\{\left(\begin{array}{cc}
\epsilon^{2} & \epsilon \\
\epsilon & 1
\end{array}\right)+k\left(\begin{array}{lll}
\epsilon^{2} & \epsilon & \epsilon \\
\epsilon & 1 & 1 \\
\epsilon & 1 & 1
\end{array}\right)\right\}
$$

It shows a good hierarchical pattern [i]

So far, we have not discussed the absolute magnitude of the neutrino mass matrix $M_{\nu}^{r a d}$. When we assume $m^{2}\left(\widetilde{e}_{R}\right) \equiv m^{2}\left(\widetilde{e}_{R 3}\right) \simeq m^{2}\left(\widetilde{e}_{R 2}\right) \simeq m^{2}\left(\widetilde{e}_{R 1}\right)$ and $m^{2}\left(\widetilde{e}_{L}\right) \equiv m^{2}\left(\widetilde{e}_{L 3}\right) \simeq$ $m^{2}\left(\widetilde{e}_{L 2}\right) \simeq m^{2}\left(\widetilde{e}_{L 1}\right)$ and the rank-1 matrix $S$ is normalized as $\operatorname{Tr}\left(S S^{\dagger}\right)=1$, the coefficient $m_{0}$ in the expression (3.16i) is given by

$$
m_{0}=\frac{1}{16 \pi^{2}} \kappa^{2} \frac{m_{1}^{(2)} m_{\tau}^{4}}{v^{2}} F\left(m^{2}\left(\widetilde{e}_{R}\right), m^{2}\left(\widetilde{e}_{L}\right)\right)
$$

where

$$
F\left(m_{R}^{2}, m_{L}^{2}\right)=\frac{1}{m_{R}^{2}-m_{L}^{2}} \ln \frac{m_{R}^{2}}{m_{L}^{2}} .
$$

If $F\left(m^{2}\left(\widetilde{e}_{R}\right), m^{2}\left(\widetilde{e}_{L}\right)\right) \simeq F\left(m^{2}\left(\widetilde{d}_{R}\right), m^{2}\left(\widetilde{d}_{L}\right)\right)$, the factor $k$ is given by $k \simeq\left(m_{b} / m_{\tau}\right)^{4}=8.6$. However, in the present paper, we regard $k$ as a free parameter. By using $1 / 16 \pi^{2}=$ $6.33 \times 10^{-3}, m_{1}^{(2)} \equiv m\left(H_{1}^{(2)}\right)=2 \times 10^{2} \mathrm{GeV}, m_{\tau}\left(m_{Z}\right)=1.75 \mathrm{GeV}, v=174 \mathrm{GeV}$ and $\tan \beta=1.5$, we obtain

$$
m_{0} \simeq 1.9 \kappa^{2} \mathrm{FeV},
$$

where $F$ is the value of $F\left(m^{2}\left(\widetilde{e}_{R}\right), m^{2}\left(\widetilde{e}_{L}\right)\right)$ in the unit of TeV. If the neutrino mass matrix $M_{\nu}$ is dominated by the radiative mass terms $M_{\nu}^{r a d}$ and we wish that the largest one of $m_{\nu i}$ is of the order of $\sqrt{\Delta m_{\text {atm }}^{2}} \simeq 0.05 \mathrm{eV}$, the value $\kappa \sim 10^{-1}$ is favorable. 


\section{Summary}

In conclusion, within the framework of an SU(5) SUSY GUT model, we have proposed a mechanism which effectively induces $R$-parity-violating terms at $\mu<m_{S B}$. In our model, those terms with lepton number violation are large enough to generate neutrino Majorana masses while those with baryon number violation are strongly suppressed so that the experimental bound of proton decay is evaded. This is related with doublet-triplet splitting. We have matter fields $\overline{5}_{L(+)}+10_{L(-)}$ and two types of Higgs fields $H_{( \pm)}$and $\bar{H}_{( \pm)}$, where $( \pm)$ denote the transformation properties under a discrete symmetry $Z_{2}$. The Higgs fields $H_{(+)}$and $\bar{H}_{(-)}$couple to $10_{L(-)} 10_{L(-)}$ and $\overline{5}_{L(+)} 10_{L(-)}$, respectively, to make the Yukawa interactions. The $\mathrm{Z}_{2}$ symmetry is only broken by the $\mu$-term, $m_{S B} \bar{H}_{(+)} H_{(-)}$, so that the $\bar{H}_{(-)} \leftrightarrow \overline{5}_{(+)}$mixing is effectively induced at $\mu<m_{S B}$. Because of the heaviness of the color triplet components of the Higgs fields, the mixing is sizable in the $S U(2)_{L}$ doublet sector, while it is negligibly small in the $S U(3)_{c}$ triplet sector.

Whether the model is harmless or not for proton decay is highly sensitive to the choice of the parameter values, especially, $m_{S B}$ and $m_{5}$. A smaller value of $m_{S B}$ gives a lighter mass for the massive Higgs fields $H_{2}$ (another one, $H_{1}$, corresponds to the Higgs field in the conventional model), so that the case spoils the unification of the gauge coupling constants at $\mu=M_{X}$. On the other hand, a large value of $m_{S B}$ induces the proton decay due to the dimension-5 operator. We have taken $m_{S B} \sim 10^{14} \mathrm{GeV}$. Also, a large value of $m_{5}$ induce the proton decay due to the exchange of squark $\widetilde{d}$. We have taken $m_{5} \sim 10^{1} \mathrm{GeV}$. Those parameter values can give a reasonable magnitude of the neutrino mass. However, the choice of such a small $m_{5}$ gives a small mixing between $\bar{H}_{(+)}$and $\overline{5}_{(+)}$, so that the case gives $\bar{U}_{22}^{(2)} \simeq \bar{U}_{11}^{(2)}$ and $\bar{U}_{11}^{(2)} \bar{U}_{33}^{(2)}-\bar{U}_{13}^{(2)} \bar{U}_{31}^{(2)} \simeq \bar{U}_{11}^{(2)} \bar{U}_{33}^{(3)}-\bar{U}_{13}^{(3)} \bar{U}_{31}^{(2)}$. Therefore, the case with $\left|\alpha^{(2)}\right| \ll 1$ cannot give a sizable deviation from $M_{d}^{T}=M_{e}$. However, this is critical for each parameter value. The details are dependent on the explicit model, i.e. on the choice of the forms $S$ and $U \equiv U_{R}^{d}$. A further careful study based on an explicit model will be required.

Anyhow, if the present scenario is working, the proton decay will be observed in the near future, because possible parameter values are in critical ranges for the proton decay in order to explain the quark and lepton (charged lepton and neutrino) masses and mixings.

The present model leads to a radiatively-induced neutrino mass matrix $M_{\nu}^{\text {rad }}$ which is given by sum of two rank-1 matrices as shown in Eq. (3.16). The "two" is originated in the two contributions from charged lepton loop and down-quark loop. This can show a good hierarchical form and hence promising.

The present model will be worth noticing. In the present model, the coupling constants $\lambda_{i j k}$ of $\nu_{L i} e_{L j} e_{R k}^{c}$ and $\nu_{L i} d_{R j}^{c} d_{L k}$ are proportional to the mass matrices $\left(M_{e}^{*}\right)_{j k}$ and $\left(M_{d}^{\dagger}\right)_{j k}$, respectively. The model will give fruitful phenomenology in flavor violating processes.

\section{Acknowledgments}

This work was supported by the Grant-in-Aid for Scientific Research, the Ministry of Education, Science and Culture, Japan (Grant Numbers 14039209, 14046217, 1474068 and 15540283). 


\section{References}

[1] Y. Koide and J. Sato, Phys. Rev. D68, 056004 (2003).

[2] T. Yanagida, in Proceedings of the Workshop on the Unified Theory and Baryon Number in the Universe, edited by O. Sawada and A. Sugamoto (KEK, Tsukuba, 1979), p. 95;

M. Gell-Mann, P. Ramond and R. Slansky, in Supergravity, edited by P. van Nieuwenhuizen and D. Freedman (North-Holland, Amsterdam,1979), p. 315; G. Senjanović and R. N. Mohapatra, Phys. Rev. Lett. 44, 912 (1980).

[3] A. Zee, Phys. Lett. 93B, 389 (1980); 161B, 141 (1985); L. Wolfenstein, Nucl. Phys. B175, 93 (1980); S. T. Petcov, Phys. Lett. 115B, 401 (1982).

[4] M. Drees, S. Pakvasa, X. Tata and T. ter Veldhuis, Phys. Rev. D57, 5335 (1998);

G. Bhattacharyya, H. V. Klapdor-Kleingrothaus and H. Pas, Phys. Lett. B463, 77 (1999);

K. Cheung and O. C. W. Kong, Phys. Rev. D61, 113012 (2000).

[5] A. Yu. Smirnov and F. Vissani, Nucl. Phys. B460, 37 (1996).

[6] A. Yu. Smirnov and F. Vissani, Phys. Lett. B380, 317 (1996).

[7] E. Witten, Phys. Lett. B105, 267 (1981);

S. Dimopoulos and F. Wilczek, NSF-ITP-82-07;

M. Srednicki, Nucl. Phys. B202, 327 (1982);

A. Masiero, D.V. Nanopoulos, K. Tamvakis and T. Yanagida, Phys. Lett. B115, 380 (1982);

E. Witten, Nucl. Phys. B258, 75 (1985);

K. Inoue, A. Kakuto and T. Takano, Prog. Theor. Phys. 75, 664 (1986);

T. Yanagida, Phys. Lett. B344, 211 (1995);

Y. Kawamura, Prog. Theor. Phys. 105, 999 (2001).

[8] K. S. Babu and S. M. Barr, Phys. Rev. D48, 5354 (1993).

[9] N. Sakai and T. Yanagida, Nucl. Phys. 197B, 533 (1982); S. Weinberg, Phys. Rev. D26, 287 (1982). For a numerical study, see T. Goto and T. Nihei, Phys. Rev. D59, 115009 (1999).

[10] L. J. Hall and M. Suzuki, Nucl. Phys. B231, 419 (1984).

[11] For example,

M. A. Díaz, J. C. Romão and J. W. F. Valle, Nucl. Phys. B524, 23 (1998);

S. Davidson and M. Losada, Phys. Rev. D 65, 075025 (2002).

[12] J. N. Bahcall, M. C. Gonzalez-Garcia and C.Penã-Garay, JHEP 0108, 014 (2001);

G. L. Fogli, E. Lisi, D. Montanino and A. Palazzo, Phys. Rev. D64, 093007 (2001); V. Barger, D. Marfatia and K. Whisnant, hep-ph/0106207; P. I. Krastev and A. Yu. Smirnov, hep-ph/0108177; SNO collaboration, Q. R. Ahmad et al., Phys. Rev. Lett. 89, 011302 (2002).

[13] KamLAND collaboration, K. Eguchi, et al., Phys. Rev. Lett. 90, 021802 (2003).

[14] Super-Kamiokande collaboration, Y. Fukuda et al., Phys. Rev. Lett. 81, 1562 (1998); M. Shiozawa, Talk presented at Neutrino 2002, Munich, Germany, May 2002 (http://neutrino.t30.physik.tu-muenchen.de/).

[15] J. Sato and T. Yanagida, Phys. Lett. B430, 127 (1998); Phys. Lett. B493, 356 (2000). 